\title{
The Effects of Social Communication Dynamics on Opinion Change
}

\author{
Niccolo Pescetelli ${ }^{1,2,3, \mathbb{D}}$ and Nick Yeung ${ }^{2}$ \\ ${ }^{1}$ Massachusetts Institute of Technology, Media Lab, 75 Amherst Street, Cambridge, MA, 02139 \\ ${ }^{2}$ Department of Experimental Psychology, University of Oxford, Anna Watts Building, Radcliffe Observatory Quarter, Woodstock Road, Oxford, OX2 6GG, United Kingdom \\ ${ }^{3}$ Max Planck Institute for Human Development, 94 Lentzeallee, Berlin, 14195
}

\begin{abstract}
Many social interactions are characterised by dynamic inter- 47 play, such that individuals exert reciprocal influence over each 48 other's behaviours and opinions. The present study investigated 49 how the dynamics of reciprocal influence affect decisions made in a social context, over and above the information communicated in an interaction. To this end, we developed a simple social decision-making paradigm in which two people are asked to make perceptual judgments while receiving information about each other's decisions. In a Static condition, information about ${ }^{54}$ the partner only conveyed their initial, independent judgment. ${ }^{5}$ However, in a Dynamic condition, each individual saw the evolv- 56 ing opinion of their partner as they learnt about and responded 57 to the individual's own judgment. The results indicated that 58 in both conditions, the majority of confidence adjustments followed a step function characterised by an abrupt change fol- ${ }^{5}$ lowed by smaller adjustments around an equilibrium, and that ${ }^{60}$ participants' confidence was used to arbitrate conflict (although 61 deviating from Bayesian norm). Crucially, interaction had sys- 62 tematic effects on opinion change relative to the Static baseline, 63 magnifying confidence change when partners agreed and reduc- 64 ing confidence change when they disagreed. These findings indicate that during dynamic interactions - often a characteristic of real-life and online social contexts-information is collectively transformed rather than acted upon by individuals in isolation. Consequently, the output of social events is not only influenced by what the dyad knows but also by predictable non-linear and self-reinforcing dynamics.

Opinion change | social interaction | confidence | decision-making Correspondence: niccolop@mit.edu
\end{abstract}

\section{Introduction}

People very often rely on others' judgments to inform their 76 decisions. We ask our family and friends what they think 77 about a particular job we are planning to apply for, the colour 78 of a new dress to buy, or the political candidate to favour. 79 When information is costly to acquire, aggregating multi- 80 ple samples of imprecise information is often more effective 81 than spending time improving a single sample (1). Aggregat- 82 ing opinions from diverse and independent sources has long 83 been known to improve judgment reliability $(2,3)$. How peo- 84 ple do this, to incorporate others' opinions and advice into 85 their decisions, has long been of central interest in social and 86 organisational psychology $(4,5)$ and more recently in com- 87 putational social science (6-8). One particularly fruitful ap- 88 proach to this question is the judge-advisor system paradigm 89 $(5,9,10)$, in which participants (the "judges") form inde- 90 pendent opinions, then are asked to revise their initial judg- 91 ment after seeing the opinion of one or more advisors. This approach provides precise control over the information provided by the advice to participants, as well as precisely defined and distinct moments at which an initial decision is formed, advice is presented, and the judge's opinion updated. Leveraging this experimental precision, previous studies have highlighted the factors affecting how personal judgments are influenced by others' opinions, like confidence $(11,12)$, power $(13,14)$, expertise $(15)$, agreement among different sources $(16)$, whether the self is involved $(17,18)$ and how distant is the the advice from the judge's own view (19-21).

However, in daily life, advisors' opinions rarely remain independent from each other or from the advisee's own judgment, as is typically implemented in judge-advisor system studies. Instead, information is collectively transformed and manipulated until it converges to a decision. For example, you might say to the friend recommending the yellow dress that you actually don't like yellow, you like green; to which she replies that she thought green was kind of nice too and when, at that point, a member of staff at the shop interrupts to say that green is going to be next year's fashionable colour, a purchase is made. Here, the processes of decision, advice and update take place all at the same time, and the line between judge and advisors blurs such that all partners affect each other's opinions without a clear distinction between cause and effect. Thus, in social contexts, interaction may happen on a continuous time scale rather than in discrete time steps, raising the possibility of non-linear dynamics.

As such, a full picture of social decision making may require analysis of interaction dynamics, moving beyond the very valuable simplification of a participant working alone as an observer of social information (cf. 22-26). Consistent with this reasoning, previous work found that individuals performing a task together become more confident and align their linguistic expressions when they are allowed face-to-face verbal interaction but not when confidence is shared without verbal interaction $(27,28)$. The finding relates back to earlier work in social psychology on "risky shifts" and group polarisation (29-31). However, little is known about the direct comparison between continuous and discrete social exchange. To address this issue, in the present study we investigated differences between static vs. dynamic interactions in social decision making, specifically in a task in which the information available to participants to make a decision was kept constant 
across interactions, thus allowing us to characterize the spe- 147 cific impact of interaction dynamics themselves, above and ${ }_{148}$ beyond the information brought to the interaction by the in- 149 dividuals involved.

In our study, participants performed simple perceptual judgements in a social scenario characterised by two people ${ }_{152}$ performing the task in parallel. On each trial, participants 153 first made private, independent judgments (about which of ${ }_{154}$ two boxes contained more dots (32)), and then were allowed ${ }_{155}$ to update their judgement, expressed on a semi-continuous ${ }_{156}$ confidence scale, based on observing the other participant's 157 opinion (on the same scale). Each participant was thus both ${ }_{158}$ judge and advisor, allowing us to monitor confidence changes 159 and influence in a system characterised by two-way infor- 160 mation exchange (22). Importantly, we compared conditions 161 characterised by static information exchange-in which par- 162 ticipants only saw their partner's initial judgment-with con- 163 ditions characterised by dynamic information exchange-in 164 which participants saw their partner's moment-by-moment 165 opinion change and thus how they themselves influenced 166 their partner's views. By keeping decision-relevant informa- ${ }_{167}$ tion constant in the two conditions, it is possible to isolate ${ }_{168}$ the contrast between static and dynamically evolving advice, 169 and thus characterise the way information is shared and col- 170 lectively transformed between advisors. Based on recent pro- 171 posals in the literature $(22,24-26)$, our key prediction is that 172 changing the directionality and dynamics of the information 173 exchange (bi-directional vs. one-way; recursive vs. static) 174 will affect the final judgments that participants will converge 175 to after social exchange, even though the perceptual evidence 176 accumulated during the private phase is the only information 177 needed to successfully accomplish the task, and this evidence ${ }_{178}$ is matched across the two conditions.

Three experiments $(\mathrm{N}=72)$ were performed. Experiment ${ }_{180}$ 1 directly compares interactive and static situations within 18 dyads. Key differences are found between the two, suggest- 182 ing that when participants are allowed to freely interact in 183 real-time, non-linearities in the confidence update are ob- 184 served. Experiments 2 and 3 (reported in Supplementary 185 Information) largely replicate the findings of Experiment 1, 186 adding controls for potential confounding factors, specifi- 187 cally that the key effects of the first study were due to fail- 188 ures in the maintenance in working-memory of one's own 189 (Experiment 2) and one's partner's (Experiment 3) opinion. 190 They also reproduce the key results using different instruc- 191 tions and a different confidence scale, thus indicating that the 192 results are robust across different settings.

\section{Methods}

Participants. Twenty-four dyads ( $\mathrm{N}=48,37$ females, age: 197 $\mathrm{M}=22.52, \mathrm{STD}=3.16$ ) were recruited in pairs for money or 198 course credits, through local announcements and the Univer- 199 sity of Oxford volunteers platform. The invitation informed 200 potential volunteers to bring a friend of the same gender. This 201 was done to avoid confounds due to gender differences in the 202 use of confidence scales and it represents standard practice in 203 the literature $(33,34)$. All dyads responded positively to the call, apart for one whose members were gender mixed due to unforeseen circumstances. The study was approved by local ethical committee. All participants gave informed consent before taking part to the study.

Paradigm. Participants sat on opposite sides of a desk divided by an occluding screen (Figure 1a), each given a separate LCD monitor, keyboard and mouse. All devices were controlled by the same computer (Dell OptiPlex 9020). All trials consisted of two stages: a private perceptual decision followed by a social exchange. During the private perceptual decision, participants performed a dot-count decision task with confidence ratings (32): Two boxes containing dots arrayed on a 20x20 grid in random order were briefly (160 $\mathrm{ms}$ ) flashed on each participant's screen to the left and right of a central fixation cross. On each trial, one box contained $200+d$ dots and the other $200-d$ dots. Participants had to indicate which box contained most dots. Task difficulty was controlled by changing the $d$ parameter and titrated to each participant independently so to reach an accuracy of around $70 \%$ (2-down 1-up procedure (35)). This ensured that independent of their individual sensitivity to the task, both experienced an equal amount of correct and error trials. Notice that, given the double staircase procedure, different dot displays were presented to the two dyad members on each trial, but the box with most dots (i.e., the correct answer) was the same for the two participants on any given trial. Thus, social information coming from the other person carried meaningful information.

Each dyad member indicated their independent response by mouse-click on a semi-continuous post-decision wagering scale (36), ranging from "100\% sure LEFT" to "100\% sure RIGHT", with the middle level removed to force participants commit to one or other decision. The scale had fifty levels per side. Participants were informed that each level of the scale corresponded to one token, which was awarded if the answer was correct and subtracted from their total score if the answer provided was wrong. Each token was worth $£ 0.01$, given to participants as cumulative earnings at the end of the experiment. Post-decision wagering scales are known to be dependent on the pay-off matrix used (37), which produces confounds if participants are too risk-seeking (38). As a control, participants were tested for loss aversion using the coin gamble task (39) and shown to be significantly loss averse (see Supplementary Information). Furthermore, we replicated the results in Experiment 2 and 3 using different confidence scale and instructions, to avoid the limitations affecting the use of post-decision wagering scales (38). Unless stated otherwise, all reported key Experiment 1 effects were replicated in Experiments 2 and 3 (complete results in Supplementary Information).

The member who responded first waited until the second had input their response. As soon as both members confirmed their answer by pressing space bar, the social exchange stage started, where each dyad member was informed about their partner's opinion. At this point, confidence changes were recorded continuously. In contrast to the standard judge- 
a

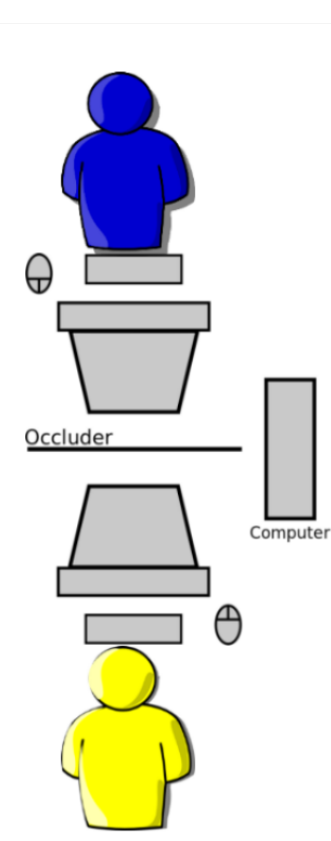

b

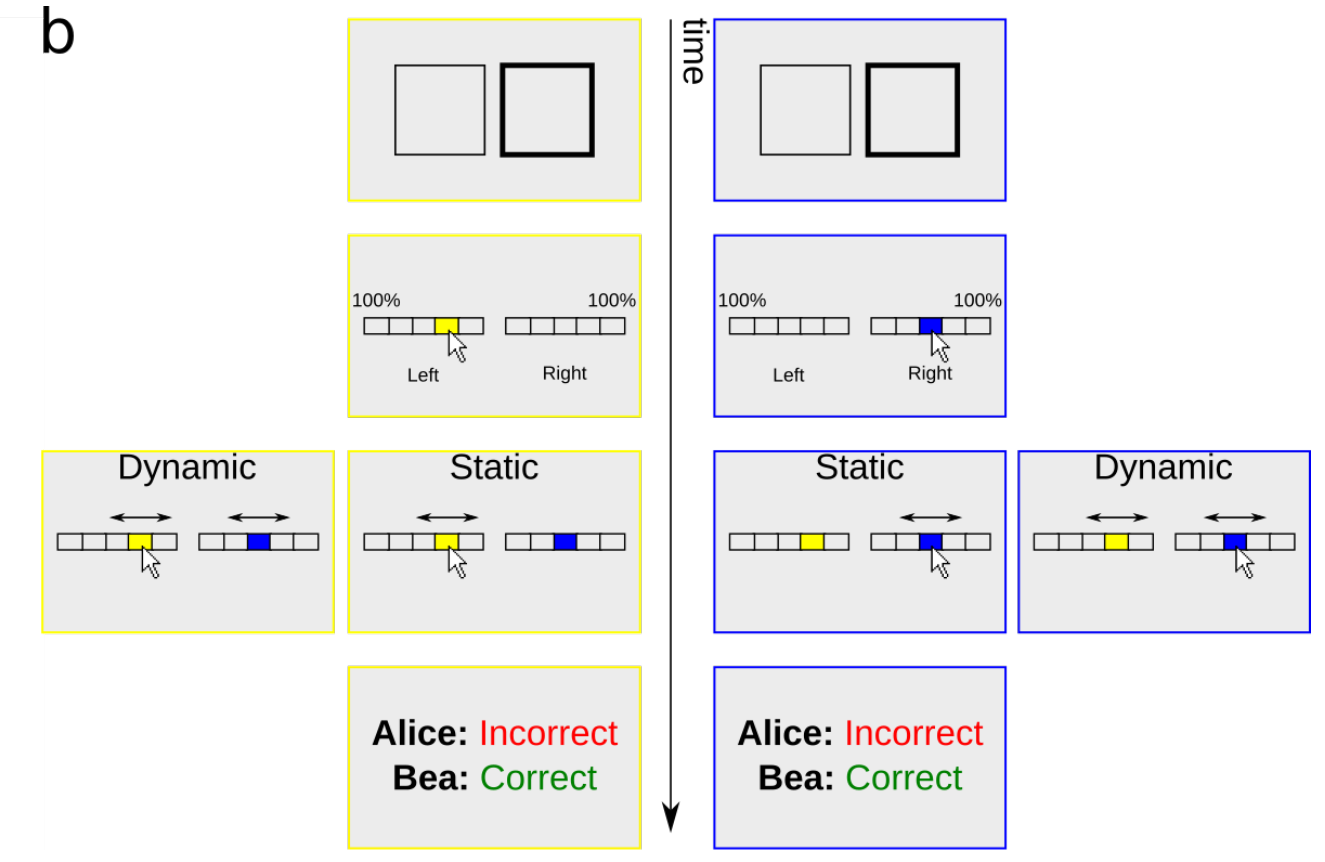

Fig. 1. (a) Participants sat on opposite sides of a wooden occluder and use one monitor, one keyboard and one mouse each, controlled by the same computer. (b) After performing a perceptual judgment independently, participants saw on their own scale their partner's initial opinion (Static condition) or their partner's evolving opinion (Dynamic condition). They received feedback on their accuracy and earnings (not shown) at the end of the trial. Bidirectional black arrows represent real-time movement along the scale. The scale used in the actual task had 50 levels per interval.

advisor system paradigm (5), where confidence updates hap- 236 pen in discrete steps, here we recorded confidence judge- 237 ments as they evolved over time: The mouse x-position along 238 the scale was recorded every $200 \mathrm{~ms}$ and each data point 239 so collected was treated as an individual post-decisional bet, 240 contributing to the total amount of tokens participants were ${ }_{241}$ supposed to maximise. This was done to incentivise partici- 242 pants to update their cursor position along the scale as soon as 243 their internal confidence changed. No clicking nor confirma- 244 tion were required during the social stage to facilitate reliable ${ }_{245}$ and continuous tracking of confidence change. This stage ex- 246 pired after five seconds (26 data points). At this point feed- 247 back was provided to both members about the tokens earned 248 by each member, and then a new trial began.

Manipulation. Our manipulation concerned only the social exchange stage. Two conditions alternated across blocks. In the Static condition, the choice and confidence level selected ${ }^{251}$ by each dyad member in the private phase appeared on their ${ }_{252}$ partner's scale as a static coloured cursor. Dyad members ${ }_{253}$ were at this point asked to continuously monitor and update ${ }_{254}$ their confidence by moving their mouse along the scale. $\operatorname{In}_{255}$ the Dynamic condition, the social exchange part started ex- 256 actly as in the Static one, with each dyad member's cursor ap- 257 pearing on their partner's scale. However, and for the whole ${ }_{258}$ duration of the social part (five seconds), if a member up- 259 dated their confidence, this would instantly appear also on 260 their partner's scale and vice-versa. This led to a situation ${ }_{261}$ where participants were not only informed of their partner's ${ }_{262}$ original opinions, but also how those opinions changed in ${ }_{263}$ real-time as a function of their own updates (Figure 1b). ${ }_{264}^{264}$ The experiment began with three blocks of practice of 10265 trials each (practice with the perceptual task alone, then in- 266 cluding the social exchange stage separately with static or dynamic interaction) followed by 14 experimental blocks of 25 trials each. Each experimental block contained 2 null trials randomly interleaved, which were private decision only trials, included so that participants were motivated to report their confidence accurately also during the private decision. In null trials, earnings were calculated from the confidence expressed during the private phase only. All other trials' earnings were computed instead from the social part. Analyses were performed to assess how social exchange (interactive or static) affected dependent variables of interest: confidence, accuracy and confidence-to-accuracy calibration. Data and analyses are available via OSF, https://osf.io/7b6py/?view_ only=793327d07511448fa58e5b757aa6a46a

\section{Results}

Confidence changes asymmetrically for consensus and condition. The use of continuous post-decision wagering gave us a time series of confidence positions for both dyad members on every trial over the five seconds time window of each trial's social part. During the social stage, participants updated their confidence over a protracted period, with the largest changes occurring about one second after first exposure to their partner's opinion (Figure 2a). Further analysis indicated that confidence updates were typically a single, unidirectional transition (towards increased confidence when partners agreed and reduced confidence when they disagreed). Thus, when we counted trial-wise transitions defined as the number of times during a trial's social stage that confidence changed from time-step $t$ to $t+1$, having remained static on the previous time-step (i.e., from $t-1$ to $t$ ) 
- we found that the number of update transitions within a ${ }_{324}$ trial significantly differed between conditions, but the effect size was small (average number of transitions: Static $=1.11$, Dynamic $=1.18, t(47)=2.95, p=.004, d=0.12)$ indicating that marginally (but consistently) more updates happened during the Dynamic condition. As the average trial consisted of a single transition, analyses on confidence reported below are performed on last confidence points registered on each trial, unless explicitly specified.

The influence that social information has on each dyad member can be quantified as the distance between their postexchange confidence and pre-exchange confidence: $\delta_{C}=$ $C_{\text {post }}-C_{\text {pre }}$, with larger absolute values corresponding to greater impact of social information. The sign of $\delta_{C}$ is expected to be positive in agreement trials, when participants typically increase their confidence, and negative in disagreement trials, when they typically decrease their confidence. A $2 \times 2$ ANOVA on confidence change (Figure 2b) with factors of consensus with respect to the initial, private decisions (disagreement vs. agreement) and condition (Static vs. Dynamic) revealed significant main effects of both consensus $\left(F(1,47)=150.26, p<.001, \eta_{G}^{2}=.7\right)$ and condition $\left(F(1,47)=9.40, p<.01, \eta_{G}^{2}=.005\right)$, but no significant interaction $(F<1)$. As expected, average $\delta_{C}$ was negative in disagreement and positive in agreement (Figure 2c). The main effect of condition indicated that participants' final level of confidence was greater in the Dynamic condition than in the Static condition, with separate paired t-tests confirming this effect held regardless of consensus: Participants increased their confidence more when in agreement $(t(47)=2.69, p=.009)$ and decreased their confidence less when in disagreement $(t(47)=2.08, p=.04)$, in Dynamic compared to Static blocks. The results indicate that opinion change is dominated by the information content conveyed (i.e., agreement vs. disagreement), with the nature of interaction (static vs. dynamic) modulating rather than fundamentally altering this pattern. Nevertheless, the observed increase in confidence in the dynamic case (averaging $1.0_{334}$ points on the scale) is non-trivial, for example, when considered in some parts of the opinion space (Figure 3) and when considering that it represents an average including trials when ${ }_{337}$ participants did not change their confidence at all (Figures $2 \mathrm{c}_{338}$ and $4 \mathrm{~b})$.

Figure 2c shows the density distribution of confidence 340 changes divided by consensus (agreement vs. disagree- 341 ment). Both agreement and disagreement distributions 342 peaked around zero, which was by far the most common ${ }^{343}$ change (note that the $\mathrm{y}$-axis in panel $\mathrm{C}$ uses a square root ${ }^{344}$ scale), indicating that very often participants ignored social 345 information. Also of note is that on some disagreement trials 346 participants actually increased their confidence, and agree- 347 ment trials they decreased it. This is a surprising result if 348 we consider that, from a normative (e.g., Bayesian) perspec- 349 tive, disagreement with an independent observer should al- 350 ways lead to reduction of confidence and agreement should ${ }_{351}$ always lead to an increase of confidence (if we assume that ${ }_{352}$ the participant believes that their partner performs better than ${ }_{353}$ chance). a

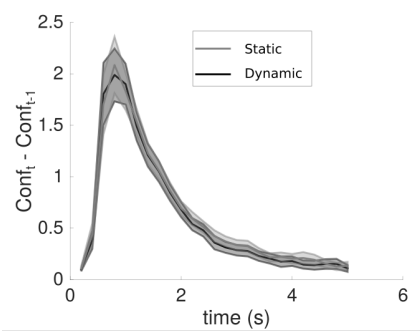

C

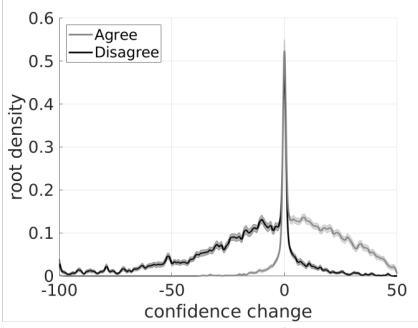

b

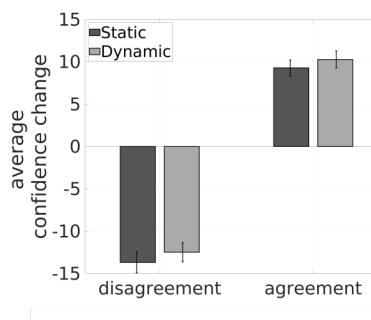

d

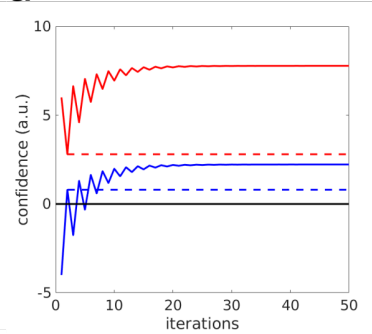

Fig. 2. (a) Average difference between confidence at time $t$ and $t-1$ across trials and for each condition. $x$-axis represents the duration of a single trial's time window during which confidence update was possible. (b) Average confidence change in agreement and disagreement trials, plotted for each condition. (c) Confidence change density distribution in agreement and disagreement. (d) Toy model emulating how confidence can increase in a Dynamic disagreement trial. In a Static condition, the agents update their initial opinion with their discounted partner's opinion (dashed lines). In Dynamic interaction, the same update rule is applied on every iteration until equilibrium is reached (solid lines).

To explore this surprising pattern of confidence change more formally, a two-way repeated measures ANOVA was computed on the probability of an irrational confidence change, defined as confidence decreases in cases of agreement and confidence increases in cases of disagreement, again with factors of consensus and condition. To avoid including trials where increases/decreases in confidence were simply due to involuntary cursor movements (a "trembling hand"), we defined a confidence "change" as a shift larger than 5 confidence points in the unexpected direction. The findings were, however, consistent across cut-offs greater than zero. Results show a significant effect of consensus $(F(1,47)=7.88, p=$ $\left..007, \eta_{G}^{2}=.07\right)$ but not of condition $(F(1,47)=2.56, p=$ $\left..11, \eta_{G}^{2}=.001\right)$ and a significant interaction between the two $\left(F(1,47)=9.90, p=.002, \eta_{G}^{2}=.005\right)$, indicating that irrational confidence changes were more frequent in disagreement than agreement ( 0.018 vs. 0.004 of trials) and that the Dynamic condition produced more frequent irrational confidence increases than the Static condition (0.020 vs. 0.015 of trials, $t(47)=2.59, p=.01)$, as well as numerically fewer irrational confidence decreases $(0.003$ vs. 0.005 of trials, $t(47)=1.98, p=.05)$. The interaction between condition and consensus was however not replicated in Experiment 2 (Supplementary Information), indicating that the result was not robust to changes in the use of the confidence scale.

Although not normatively prescribed (e.g., in a Bayesian framework), opinion aggregation strategies described in the literature $(3,40,41)$ can explain irrational decreases in confidence in cases of agreement. For example, averaging of 
confidence would lead to this outcome when a partner agrees 411 but is much less confident than the participant, such that ${ }_{412}$ the participant concludes that they should not have been so confident in the first place. Of more interest, there-413 fore, are irrational increases in confidence after disagree- 414 ment, which occurred more frequently than irrational de- 415 creases but which are difficult to reconcile with any obvious 416 confidence-update strategy. Notably, this irrationality could 417 occur through non-linear dynamics introduced by real-time 418 interaction, as explored in a simple simulation of belief up- 419 date in the two conditions. Consider an example trial in 420 which a participant $\left(P_{\max }\right)$ starts off on a confidence level ${ }^{421}$ of $C_{\max }^{\text {pre }}=0.6($ a.u. $)$ while their partner $\left(P_{\min }\right)$ weakly dis- 422 agrees $\left(C_{\min }^{\text {pre }}=-0.4(\right.$ a.u. $\left.)\right)$. Suppose next that both partic- 423 ipants use a simple update strategy, namely summing their 424 own initial confidence with their partner's weighted confi- 425 dence (here: weight $=0.80$ ). This strategy has been shown ${ }^{426}$ elsewhere to be a good approximation of confidence change 427 in joint decisions (40). In a situation where no interaction 428 is allowed, participants can only use their partner's initial ${ }^{429}$ opinion to get to a new confidence, thus reaching levels of 430 $C_{\max }^{\text {post }}=0.28$ and $C_{\min }^{\text {post }}=0.08$. However, a different out- 431 come is observed in the interactive scenario in which the par- 432 ticipant has access at each iteration to the partner's current ${ }^{433}$ confidence level and uses it to recursively updates his/her 434 initial confidence. Figure $2 \mathrm{~d}$ shows that this simple strat- 435 egy leads to an oscillatory update that stabilises for $P_{\max }{ }^{436}$ on a higher confidence than initially held. The effect reflects 437 the fact that if $P_{\min }$ crosses the decision boundary 0 , dis- 438 agreement turns into agreement, thus supporting $P_{\max }$ 's ini- ${ }^{439}$ tial opinion, instead of providing contradictory evidence, and 440 therefore leads to an increase in confidence despite initial dis- 441 agreement.

To test for recursive dynamics in our behavioural data we counted, for each condition, the average number of vacil- 444 lations in a trial, namely the number of times the direction 445 of the update (i.e., stationary/increase/decrease) changed in 446 the update window. Formally: $V_{i}=\left(r_{t}-r_{t-1}\right) \neq 0$, with ${ }_{447}$ $r=\operatorname{sign}\left(C_{t}-C_{t-1}\right)$, for each trial $i$. Across participants, 448 the average number of vacillations in a trial was significantly 449 more frequent in the Dynamic than Static condition, provid- 450 ing some support for the intuition behind our simple model, ${ }_{451}$ although the effect was a small one (Static $=2.41 \pm 1.26,{ }_{452}$ Dynamic $=2.55 \pm 1.27, t(47)=2.62, p=.01, d=0.11)$. In ${ }_{453}$ this way, irrational increases in confidence in cases of dis- 454 agreement could arise if participants updated their judgments 455 based on the observed updates of their partner (which in turn ${ }_{456}$ are influenced by their own judgment), thus treating these 457 updates as if they were independent evidence. A predic- 458 tion arising from this simulation is that confidence increases 459 in disagreement should only be observed for the participant 460 expressing higher confidence in their judgment on the trial ${ }_{461}$ (the dominant individual). Indeed, participants showed a sig- 462 nificantly higher rate of irrational increases in the Dynamic ${ }_{463}$ condition compared to a Static condition when they were the ${ }_{464}$ more confident of the partners on the trial (0.012 vs. 0.008 of 465 disagreement trials, $t(47)=3.29, p=.001, d=.21$ ), and not ${ }_{466}$ when less confident ( 0.007 vs. 0.007 of disagreement trials, $p>.8)$.

Dyadic interactions in opinion space. The preceding analyses explore opinion change when the data are aggregated across broad categories (e.g., agreement vs. disagreement trials). To explore more nuanced behaviour as a function of participants' absolute and relative levels of confidence, we explored our data as a function of a 2-dimensional "opinion space" (40) as shown in Figure 3. The figure plots confidence change following interaction within this space. Here, the $\mathrm{x}$-axis value indicates the confidence of whichever participant in the dyad is the more confident on any given trial in the private judgment phase, henceforth the "dominant" member $\left(P_{\max }\right)$ on the trial, thus ranging from 1 (minimum confidence) to 50 (maximum confidence). The y-axis value gives the confidence of the less confident, or "dominated", member of the dyad in their initial judgment on the trial $\left(P_{\min }\right)$, on a scale ranging from -50 (disagreement with maximum confidence) to 50 (agreement with maximum confidence). This plot creates a grid of possible social situations in which the dyad's state - both members' choices and their confidence - is fully represented by $(x, y)$ coordinates, while collapsing across the particular side of participants' choices (left vs. right box). In Figure 3, pixel colour indicates the median change in confidence from pre- to post-exchange of the dominant (upper panels) and dominated (lower panels) member of the dyad on each trial. The dominant and dominated participants' confidence change can be combined into a single vector field (Figure S1) visualizing dyadic transitions in opinion space (40). Real-time animations of confidence transition in opinion space for each condition can be found at https://osf.io/7b6py/?view_only= 793327d07511448fa58e5b757aa6a46a.

Figure 3 shows once again the overall increase in confidence seen in dynamic vs. static interactions, with dynamic interaction characterised by greater increases in confidence when partners agreed (cf. larger red area in upper half of Figure $3 b$ than $3 a$ ) and smaller decreases when they disagreed (cf. smaller blue area in lower half of Figure $3 \mathrm{~b}$ than 3a) The contrast plots (Figure $3 \mathrm{c}$ and $3 \mathrm{f}$ ) more precisely identify the locus of these between-condition differences, while also highlighting the magnitude of the effects in certain conditions. Thus, dynamic interaction leads to particularly marked confidence increases when partners began the social stage in agreement but with low confidence (points marked $x$ in Figure 3). Confidence change in the Dynamic condition (panel b,e) in these conditions of uncertain agreement is $20-30$ confidence points, and $\sim 15$ points greater than in static interaction blocks: Thus, when interacting dynamically, but not statically, two uncertain partners tended to reinforce each other's opinion so that together they converged on the maximum possible confidence level. The other key point of interest in the contrast plots lies in the disagreement half of the opinion space, specifically at the points labelled $y$ in Figure $3 \mathrm{c}$ and $3 \mathrm{f}$. These were trials in which the dominant member was highly confident and the dominated mem- 

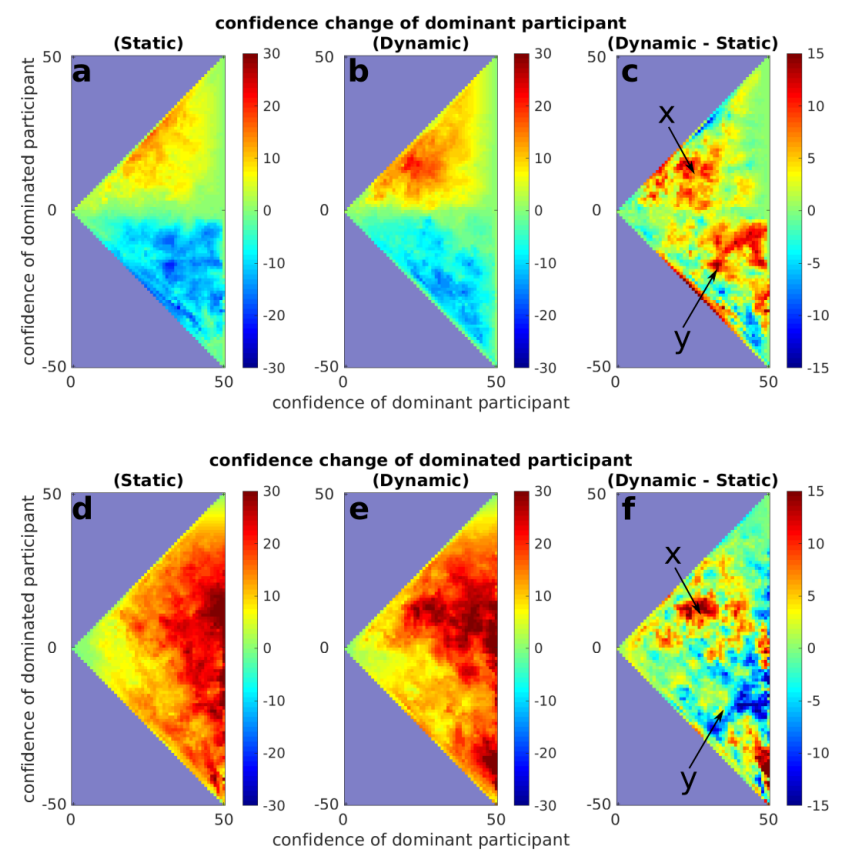

Fig. 3. Confidence change observed for every social situation, according to the confidence of the more $\left(P_{\max }\right)$ and less $\left(P_{\min }\right)$ confident participant. $(\mathrm{x}, \mathrm{y})$ coordinates represent the pre-exchange dyad state, while the colour (z-axis), represents ${ }^{515}$ confidence change. Panels a-c represent confidence change in dominant trials in 516 Static condition, Dynamic condition and their contrast respectively. Panels d-f represent confidence change in dominated trials in Static condition, Dynamic condition ${ }^{517}$ and their contrast respectively. Panels $\mathbf{c}$ and $\mathbf{f}$ represent contrasts between the two ${ }^{518}$ conditions (contrasts $\mathbf{b}$-a and $\mathbf{e}-\mathbf{d}$ respectively). Trials within each condition and 519 dyad were linearly interpolated, due to data sparseness.

ber weakly disagreed, a situation described in our simple simulation above: The warmer colour at $y$ in Figure $3 \mathrm{c}$ indicates that disagreement had markedly less impact on the dominant partner's confidence when the dyad interacted dynamically rather than statically. The corresponding point in Figure $3 \mathrm{f}$ indicates that, similarly, larger shifts in the dominated participant's confidence toward the dominant individual's posi- ${ }^{527}$ tion were observed in Dynamic compared to Static blocks. Overall, therefore, this opinion space analysis identifies the conditions under which dynamic interaction has its largest impact - when partners agree with symmetric low levels of confidence, and when they disagree with asymmetric levels of confidence-and shows that this impact is substantial in ${ }_{530}$ these conditions.

Comparing confidence updates with Bayesian aggregation. We next compared participants' confidence changes in interaction with a normative Bayesian strategy. For this analysis, we treated confidence ratings as subjectively estimated probabilities that a particular decision is correct (42-44), while ${ }^{536}$ applying a linear transformation to prevent values of 0 and $1^{537}$ and thus avoid impossible solutions: range of $0.01=" 100 \%$ Sure LEFT" to $0.99=$ "100\% Sure RIGHT"). The probabilities $p_{s}$ and $p_{p}$ so obtained-representing dyad members' independent priors on RIGHT being correct (cf. 45) — can now be integrated into the normative posterior:

$$
\text { post }_{\text {norm }}=\frac{p_{s} p_{p}}{p_{s} p_{p}+\neg p_{s} \neg p_{p}}
$$
served decision biases of egocentric discounting and confirmation bias that affect the perception of social information $(10,19,46)$. The main effect of interaction condition mirrored the overall effect of dynamic interaction leading to increased confidence, thus increasing the discrepancy from optimal updating in case of disagreement, but reducing it in case of agreement.

Bayes theorem can also be used to infer the participants' perception of the social information. Equation 1 is used by the optimal observer to infer the predicted posterior confidence given a prior confidence level $p_{s}$ and a partner's opinion $p_{p}$. However, by solving the equation for $p_{p}$ (i.e., the likelihood term), we can infer the perceived partner's confidence $\hat{p}_{p}$, given the degree to which the participant updated their confidence from their initial judgment (the prior, $p_{s}$ ) to a final decision following social interaction (the posterior, post $_{s}$ ):

$$
\hat{p}_{p}=\frac{\operatorname{post}_{s}\left(p_{s}-1\right)}{2 p_{s} \text { post }_{s}-p_{s}-\text { post }_{s}} ;
$$

In this way we can quantify the weight that participants assigned to their partner's judgement across trials, contrasted with the weight (i.e., probability correct) that the partner themselves conveyed in their confidence judgments. Comparing the distribution of these values across trials reveals how objective social information (i.e., partner's stated support for the participant's opinion) becomes distorted when perceived and acted on by the participant (i.e., actual use of partner's social information). As shown in Figure $4 \mathrm{~b}$, whereas partners' stated confidence ratings were fairly evenly distributed in terms of conveying social information ranging from maximal disagreement ( 0 on the $\mathrm{x}$-axis) to maximal agreement (1), the weight that participants assigned to their partners views showed almost categorical behaviour, with separate peaks at $\hat{p}_{p} \sim 0.5$ (i.e., advice treated as carrying little or no information) and $\hat{p}_{p} \sim 1.0$ (i.e., advice treated 


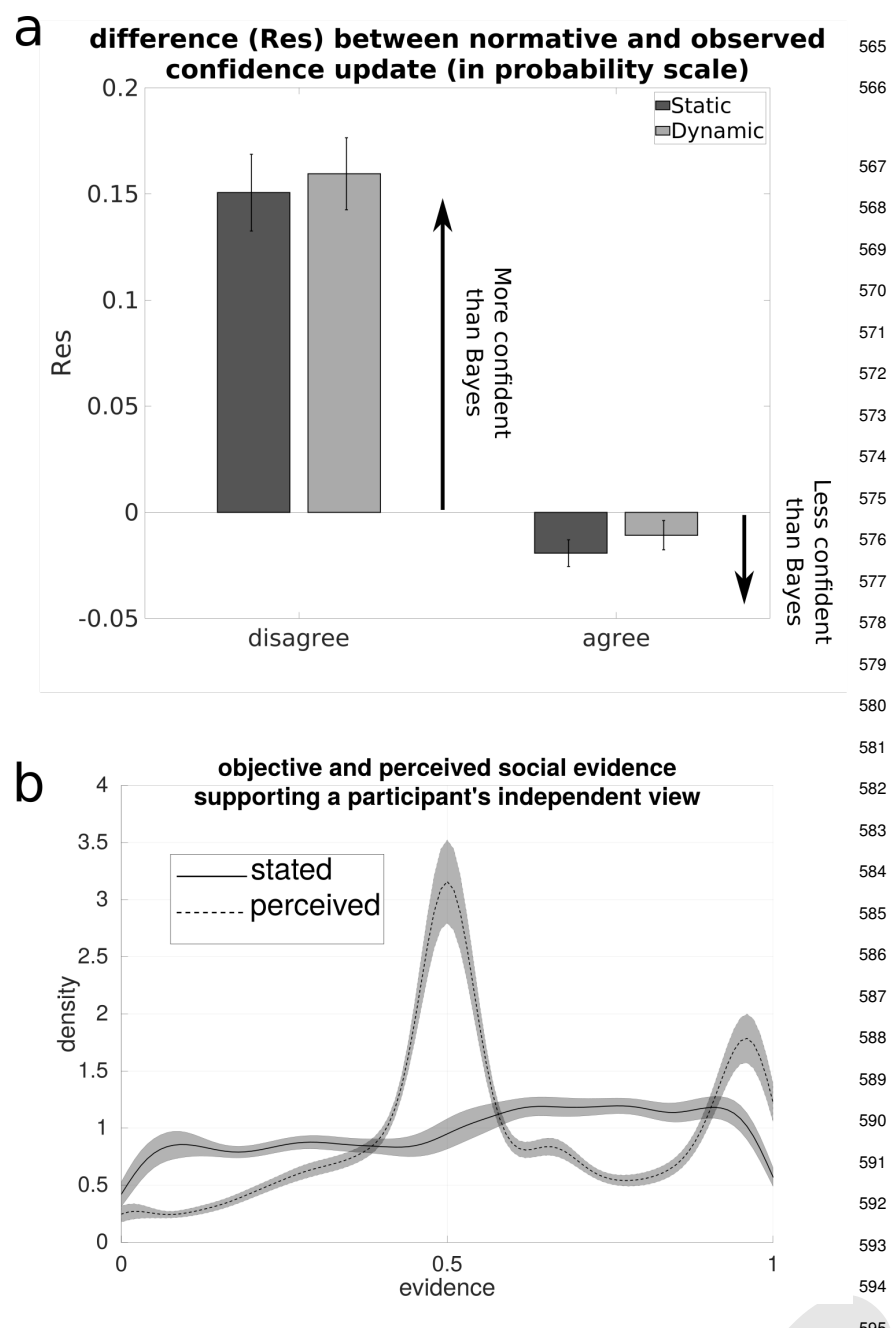

Fig. 4. (a) Difference Res between observed confidence change and normatively prescribed confidence change. (b) Contrast between partner's stated support for ${ }^{5}$

\section{.} the participant's independent view (objective social evidence) and the participant's 597 perceived support of the partner's opinion (perceived social evidence), as inferred 598 using inverted Bayes. The plots show density distributions calculated with a Gaussian kernel method (bandwith=0.04). A value of 1 (a value of 0 ) corresponds to ${ }^{599}$ social information that maximally agrees (disagrees) with one's initially expressed 600 opinion.

as objectively correct) (47). Further analysis (Supplemen- ${ }_{603}$ tary Analysis) confirmed that in both interactions conditions, 604 and both when holding the dominant or dominated view, par- ${ }_{605}$ ticipants tended to use partner's objectively stated opinion 606 in a self-serving manner, thus overplaying the support that ${ }_{607}$ social information information provided for one's own ini- 608 tial judgment. To formally test for this distortion, agree- 609 ment trials (i.e., $x$-axis greater than 0.50 ) were divided into ${ }_{610}$ six bins of evidence, and a linear model with a linear and quadratic terms was fitted: frequency $=\beta_{0}+\beta_{1}$ evidence $+{ }_{611}$ $\beta_{2}$ evidence $^{2}$. Results showed that the quadratic terms was 612 not significant for partner's stated evidence (dominant: $\beta_{2}={ }_{613}$ $0.50, S E=0.21$; dominated: $\beta_{2}=0.22, S E=0.46$ ), but ${ }_{614}$ was significant for the participant's perceived evidence (dom- 615 inant: $\beta_{2}=2.39, S E=0.57, t=4.19, p=.02$; dominated: 616 $\beta_{2}=3.84, S E=0.56, t=6.75, p=.006$ ), explaining $80 \% 617$ and $90 \%$ of the variance for dominant and dominated trials 618 respectively (Adjusted $R^{2}$ ). The result is evidence of the fact ${ }_{619}$ that participants tended to treat advice bimodally as either ${ }_{620}$ highly supportive of their own initial opinion or as carrying little informational value.

Coupling of confidence changes during interaction. The analyses above consider opinion change for each dyad member separately. To investigate how the magnitude of partners' opinion changes co-varied across trials, we calculated across-trial correlations of absolute confidence change, $\left|\delta_{C}\right|$, for members of each dyad. Pearson's $r$ coefficients were calculated for each dyad as a function of dyadic consensus (agree vs. disagree) and interaction condition (dynamic vs. static). A $2 \times 2$ ANOVA on the resulting values across dyads (Figure 5) revealed a main effect of consensus $(F(1,22)=20.93, p<.001)$ but not of condition $(F(1,22)=$ $1.71, p=.20)$, and a significant interaction between the two $(F(1,22)=38.39, p<.001)$. When dyad members did not see each other's confidence changes in the social exchange stage (Static condition), confidence changes did not correlate significantly between partners $\left(h_{1}=r>0, t(22)<1.28, p>\right.$ $.2, d=0.26$ ). This finding is not unexpected, but nor is it trivial-for example, a positive correlation might be expected in agreement trials as a consequence of a boost to both participants' confidence when they agreed but were initially uncertain, such that agreement led to increased confidence for both. This did not seem to occur. In contrast, in the Dynamic condition, partners' confidence changes became coupled: In agreement trials, the correlation was positive, indicating that the more one member increased their confidence, the more their partner also increased their confidence. In disagreement, the correlation was negative, indicating that the more one member decreased their confidence in their initial decision, the less the partner decreased theirs. Pairwise contrasts showed that, compared to Static condition, in the Dynamic condition correlation coefficients were significantly greater for agreement $(t(22)=4.89, p<.001, d=1.20)$ and somewhat smaller for disagreement $(t(22)=-2.02, p=.05, d=$ $-0.52)$. The negative correlation found in disagreement Dynamic trials, was replicated in Experiment 3, but not in Experiment 2 (Supplementary Information). Further analyses (Supplementary Information) showed that the effect could not be explained by participants using their partner's reaction times (i.e., "unwillingness to move") in the Dynamic but not in the Static condition. Coupling of confidence changes in interaction suggests that participants were influenced by their partner's confidence change when updating their own opinion, rather than basing their change solely on their partner's initial (independent) judgment as normatively prescribed.

Performance analysis. A tenet in the judgment aggregation literature is that social interaction hampers collective wisdom by breaking the independence of the individual judgments (48). The traditional interpretation of the wisdom of crowds $(2,49)$, named here the "noise cancelling hypothesis", explains the accuracy improvement observed in opinion aggregates as a statistical phenomenon where noise reduces after independent samples (here the private initial opinions) are averaged together. This hypothesis predicts that breaking the independence between measures should have negative ef- 


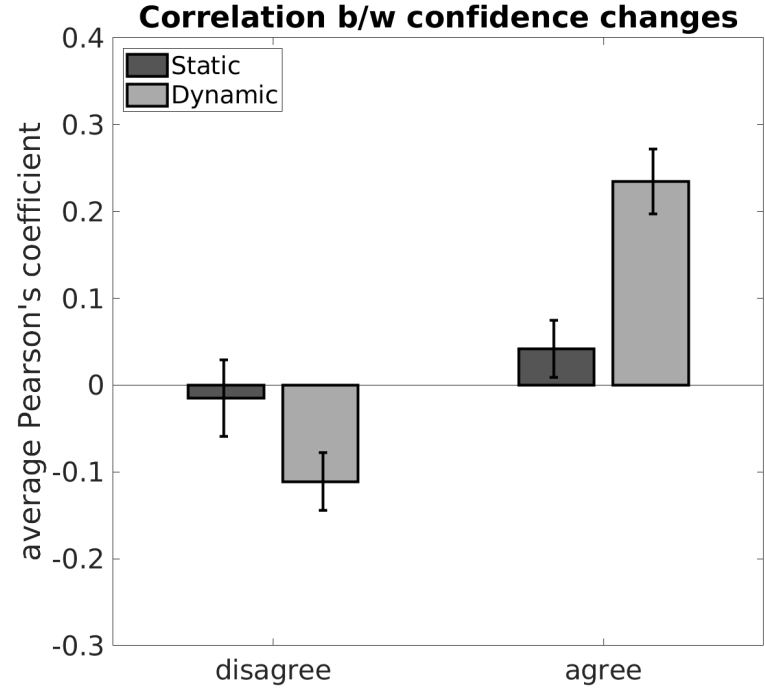

Fig. 5. Effect of condition on the correlation between absolute confidence chang of the two participants across trials. Average Pearson's correlation coefficient is plotted as a function of consensus and condition. One dyad removed for a missing ${ }^{674}$ cell.

fects on accuracy, as errors become correlated instead of av- 677 eraging out. In their experiment, Lorenz and colleagues (48) 678 showed that simple exposure to others' opinions had dam- 679 aging effects on aggregate accuracy. If the noise cancelling 680 interpretation is correct, in the present study we should ob- ${ }_{681}$ serve that (1) simple exposure to another person's opinion ${ }_{682}$ negatively affects performance; (2) the effect of social expo- 683 sure is even more damaging on performance in the Dynamic ${ }_{684}$ condition, as this condition affects the independence of the 685 individual estimates more than the Static one (Figure 5).

A 2-way ANOVA on accuracy with factors condition (Static ${ }_{68}$ vs. Dynamic) and decision stage (pre- vs. post-exchange) ${ }_{688}$ showed a significant effect of decision stage $\left(F(1,47)={ }_{689}\right.$ $\left.47.00, p<.001, \eta_{G}^{2}=.16\right)$ but no significant effect of con- 690 dition $(F<1)$ nor a significant interaction $(F<1)$. Social ${ }_{691}$ information had a beneficial effect on average accuracy (pre- 692 exchange accuracy $=0.72$, post-exchange accuracy $=0.75)$. 693 The finding indicates that, contrary to (48), exposure to an- 694 other person's opinion did not reduce accuracy. Furthermore, ${ }_{995}$ Dynamic interaction did not reduce accuracy improvement 696 compared to Static, indicating that increased dependence be- 697 tween confidence updates (as indicated in the analyses of Fig- 698 ure 5) had no significant damaging effect on accuracy (or ac- 699 curacy improvement) either. However, condition might have affected people's opinions 70 without making them changing their initial answer (5). We 702 thus tried to define a more nuanced definition of accuracy 703 improvement as confidence changes toward or away the cor- 704 rect end of the scale: $\delta_{a c c_{G}}=\left(C_{\text {post }}-C_{\text {pre }}\right)^{A c c} *\left(C_{\text {pre }}-705\right.$ $\left.C_{\text {post }}\right)^{1-A c c}$, where $A c c \in\{0,1\}$. We tested whether partic- 706 ipants improved their initial judgment although keeping their 707 initial views. The same $2 \times 2$ ANOVA did not show any ef- 708 fect of condition on graded accuracy improvement $\delta_{\text {acc }_{G}}$ nor 709 significant interaction $(F<1)$, thus rejecting the idea that 710 condition had an influence on accuracy.
A third and final measure of performance considered was the calibration of confidence relatively to objective accuracy, defined here as the type II $A_{R O C}$ (50). The same twoway ANOVA used for choice accuracy was run on type II $A_{R O C}$. Results show a significant effect of decision stage $\left(F(1,47)=89.58, p<.001, \eta_{G}^{2}=.25\right)$, indicating calibration improvement from pre- to post-exchange phase (0.60 vs. $0.66)$, but no effect of condition nor interaction between the two $(F<1)$, further confirming that, overall, the nature of interaction (static vs. dynamic) did not impact on calibration or improvement thereof.

\section{Discussion}

The present study compared social exchange involving static, one-step communication with exchange characterised by dynamic and recursive interaction. We hypothesized that realtime bi-directional dynamics, which characterise many daily life interactions of social influence (22), would have systematic impact on decisions made in a social context, over and above the effects of the information brought by each individual to the interaction, as studied in traditional judge-advisor system experiments $(5,51)$. Across conditions with equal information available - because in both the Dynamic and Static conditions, dyad members viewed perceptual evidence separately and for $160 \mathrm{~ms}$ only-we observed different opinion aggregation strategies according to the nature of communication between partners. Specifically, dynamic interaction produced higher confidence changes in agreement and smaller confidence changes in disagreement by breaking the independence of dyad members' opinions: Confidence changes of the two participants became correlated during Dynamic interaction compared to a Static baseline so that, in agreement, greater increases in confidence for one member were associated with greater confidence increases for their partner, leading to belief escalation. In disagreement, greater changes in confidence for one member were associated with smaller changes in confidence for their partner, reducing the impact of disagreement on belief updating.

These combined results can be understood in terms of individuals making use of their partner's change in confidence to update their opinion, without taking into account that this change was biased (and indeed generated) by exposure to their own judgment. People are known to quickly reach decisions even when information is scarcely available by taking into account a host of circumstantial variables that are known to co-vary with problem-specific evidence, but that are not themselves strictly task-relevant (52-54). Interpreting someone's changes of mind as another cue for confidence is sensible in many daily life situations. Indeed, if somebody's beliefs are fickle, we have reasons to believe s/he must be uncertain. In the case of social interaction, however, this heuristic leads to potentially sub-optimal self-reinforcing dynamics, when a person uses the impact of his/her own opinion on the other person as evidence for the opinion itself. The impact of this circular reasoning was particularly marked in the case of two partners agreeing but with low or intermediate levels of confidence: In these cases, dyads often escalated together to- 
wards maximal confidence in their shared judgement through 768 dynamic interaction, an effect not observed when information 769 exchange was static and limited to each individual's indepen- 770 dent judgment.

Phenomena of belief escalation have already been observed ${ }^{772}$ in relation to sharing confidence judgments in group deci- ${ }^{773}$ sions $(27,55)$. These studies found that reciprocal social in- ${ }^{774}$ teraction can make people's judgments more risky and ex- ${ }^{775}$ treme, a result reminiscent of earlier findings on group po- ${ }^{776}$ larisation (29). The present study shows that a crucial cause ${ }^{777}$ of confidence escalation is the use of redundant information: ${ }^{778}$ People should only use each other's independent opinions to ${ }^{779}$ arrive at a final decision, because this is the only informa- ${ }^{780}$ tion that carries task-relevant value. However, they also (in- ${ }^{781}$ correctly) use how much their own opinion is affecting their ${ }^{782}$ partners. This strategy creates dependencies that can poten- ${ }^{783}$ tially create unwanted non-linear dynamics. Beyond confi- ${ }^{784}$ dence escalation as described above, a more subtle but nev- ${ }^{785}$ ertheless consistent effect of this strategy in our data was that ${ }^{786}$ participants occasionally increased their confidence despite ${ }^{787}$ initially learning that their partner disagreed with them (a sur- ${ }^{788}$ prising irrationality that occurred more frequently than the ${ }^{789}$ mirror effect of decreasing their confidence when a partner ${ }^{790}$ agreed). Our analyses suggest that partner's changes of mind ${ }^{791}$ are sometimes perceived as supporting evidence for one's ${ }^{792}$ views: When a partner initially disagrees, but subsequently ${ }^{793}$ loses confidence in this view (or even reverses it) on learning ${ }^{794}$ the participant's view, it seems that the participant takes this as evidence in favour of their initial position, and therefore increase their confidence. This phenomenon cannot easily be explained by static aggregation rules like averaging, summing or Bayesian integration $(3,40,41)$, but is predicted by a recursive update model.

Notwithstanding these differences in confidence updating 799 across static vs. dynamic interactions, participants' overall 800 accuracy showed a consistent benefit from social information 801 exchange. The benefits of social exchange were of similar 802 magnitude across static and dynamic interaction conditions, 803 whether this benefit was measured in terms of overall ac- 804 curacy, confidence in the correct answer, or the calibration 805 between confidence and objective performance. This parity 806 was observed despite different interaction dynamics across 807 conditions, at least in part because these dynamics led to op- 808 posing effects on accuracy across trials. Thus, when we con- 809 trast participants' behaviour with a Bayes optimal strategy, 810 we find that they consistently underweighted their partners 811 opinion and thus did not benefit optimally from social ex-812 change. When comparing dynamic to static interaction, this 813 underweighting relative to Bayes was greater in cases of dis- 814 agreement and reduced in cases of agreement, for the reasons 815 discussed above, resulting in little net difference in accuracy 816 across conditions. An interesting issue for future is whether 817 the introduction of systematic biases across members' initial 818 judgments (e.g., 40) might generate greater errors in interac- 819 tion than non-interaction, due to escalation of confidence in 820 incorrect answers (56-58).
Although it is informative to compare empirically observed behaviour with an optimal model grounded in a probabilistic interpretation of confidence (45), participants clearly departed from the Bayesian strategy commonly explored in the opinion aggregation literature $(41,59,60)$, in which opinions are combined in relation to expressed confidence. The use of inverse Bayes allowed us to compare the objective information conveyed from social partners with the information perceived by a participant. Results evidenced that social information was distorted by the receiver in a self-serving manner and asymmetrically for agreement and disagreement, in line with the advice-taking literature suggesting the presence of egocentric and confirmation biases $(10,46)$. More specifically, the weighting of partner's information (i.e., use of social information) seemed to follow a bimodal distribution, with peaks around $50 \%$, corresponding to neutral social information, and $100 \%$, corresponding to maximally supporting social information. These results are consistent with the hypothesis that people are solving a categorical inference problem (cf. 47): Instead of using continuous social information as it is provided by their social partners, participants seem to classify each trial as "partner is wrong" vs. "partner is correct" and, once this categorization is performed, use social information accordingly to update their views. Accordingly, participants would try to minimize situations of uncertainty (e.g. 0.25 or 0.75 evidence), thus maximizing the impact of social information on final confidence.

Our key experimental results were replicated using linearmixed effects regressions and by running two further experiments, reported in the Supplementary Information material. The same paradigm used in Experiment 1 was extended with two aims: (a) to replicate the results found in the current experiment using a different method to report confidence, and (b) to make sure that the escalation effects found were not due to confounding factors. Indeed, a perhaps more parsimonious explanation of the interactive effects found is that participants quickly forgot the initial configuration and were only left with their current expressed opinions. This hypothesis can, for example, explain non-linear opinion reinforcement. We therefore included confidence anchors to remind each participant of the initial, independently expressed confidence in their own judgment (Experiment 2) and from their partner (Experiment 3). Replication of key findings across experiments-including confidence change asymmetry, update coupling, and confidence escalation-indicated that the results are robust to different instructions and different uses of the confidence scale. Collectively, these findings show that social influence depends not only the task-relevant information provided by social partners, but also on the modality in which information is shared and transformed across individuals. Real-time interaction, as seen in most daily social exchanges, is recursive and dynamic in nature, and thus an understanding of social decision making requires consideration of these phenomena. 


\section{Conclusion}

In many daily life scenarios, information is collectively ma- 897 nipulated by several agents who interact in closed-loop recur- ${ }_{899}^{898}$ sive dynamic systems. Here we explored these dynamics in 900 a controlled setting, using a simple perceptual decision task ${ }_{902}^{901}$ and computer interface for communication, to allow quantifi- 903 cation of the impact of social exchange on individual decision ${ }_{905}^{904}$ making. We find that interaction dynamics affect individuals' ${ }_{906}$ decisions above and beyond the content of their shared infor- ${ }_{908}^{907}$ mation-the medium becomes (part of) the message. In the 909 aggregate, the impact of interaction dynamics is subtle but ${ }_{911}^{910}$ consistent, evident as a general increase in confidence in de-912 cisions made. However, the impact is very marked in specific ${ }_{914}^{913}$ situations, notably when shared but uncertain beliefs become 915 mutually reinforced to a state of near certainty, and when $a_{917}^{916}$ decision maker interprets vacillation in a partner's weak dis- 918 agreement as positive evidence for their views.

\section{ACKNOWLEDGEMENTS}

Suport of the Clarendon Fund the Christ Church Cole and the Department of Experimental Psychology of the University of Oxford.

\section{Bibliography}

Edward Vul, Noah Goodman, Thomas L. Griffiths, and Joshua B. Tenenbaum. One and 929 Done? Optimal Decisions From Very Few Samples. Cognitive Science, pages 1-39, 1930 2014. ISSN 03640213. doi: $10.1111 / \operatorname{cogs} .12101$.

2. Francis Galton. Vox Populi. Nature, 75(1949):450-451, 3 1907. ISSN 0028-0836. doi: 932 10.1038/075450a0.

3. Richard P. Larrick and Jack B. Soll. Intuitions About Combining Opinions: Misappreciation 934 of the Averaging Principle. Management Science, 52(1):111-127, 1 2006. ISSN 0025-1909. 935 doi: $10.1287 / \mathrm{mnsc} .1050 .0459$

4. Stephen E. Humphrey, John R. Hollenbeck, Christopher J. Meyer, and Daniel R. Ilgen. 937 Hierarchical team decision making. In Personnel and Human Resources Management, 938 pages 175-213. Emerald Group Publishing Limited, 2002. ISBN 0762309733. doi: 10. 939 1016/S0742-7301(02)21004-X.

5. Silvia Bonaccio and Reeshad S. Dalal. Advice taking and decision-making: An integrative 941 literature review, and implications for the organizational sciences. Organizational Behavior 942 and Human Decision Processes, 101(2):127-151, 11 2006. ISSN 07495978. doi: 10.1016/943 j.obhdp.2006.07.001.

6. Joshua Becker, Devon Brackbill, and Damon Centola. Network dynamics of social influ- 945 ence in the wisdom of crowds. Proceedings of the National Academy of Sciences, page 946 201615978, 6 2017. ISSN 0027-8424. doi: 10.1073/pnas.1615978114.

7. Albert B. Kao, Andrew M. Berdahl, Andrew T. Hartnett, Matthew J. Lutz, Joseph B. Bak- 948 Coleman, Christos C. Ioannou, Xingli Giam, and lain D. Couzin. Counteracting estimation 949 bias and social influence to improve the wisdom of crowds. Journal of The Royal Society 950 Interface, 15(141):20180130, 4 2018. ISSN 1742-5689. doi: 10.1098/rsif.2018.0130. 951

8. Matthew J Salganik, Peter Sheridan Dodds, and Duncan J. Watts. Experimental Study of 952 Inequality and Unpredictability in an Artificial Cultural Market. Science (New York, N. Y.), 311953 (854):854-856, 2006. doi: 10.1126/science.1121066.

9. Janet A. Sniezek and Lyn M. Van Swol. Trust, Confidence, and Expertise in a Judge-Advisor 955 System. Organizational behavior and human decision processes, 84(2):288-307, 3 2001. 956 ISSN 0749-5978. doi: 10.1006/obhd.2000.2926.

10. Ilan Yaniv and Eli Kleinberger. Advice Taking in Decision Making: Egocentric Discounting 958 and Reputation Formation. Organizational behavior and human decision processes, 83(2): 959 260-281, 11 2000. ISSN 0749-5978. doi: 10.1006/obhd.2000.2909.

11. Paul C. Price and Eric R. Stone. Intuitive evaluation of likelihood judgment producers: ev- 961 idence for a confidence heuristic. Journal of Behavioral Decision Making, 17(1):39-57, 1962 2004. ISSN 0894-3257. doi: 10.1002/bdm.460.

12. Sunita Sah, Don A Moore, and Robert J Maccoun. Cheap talk and credibility: The conse- 964 quences of confidence and accuracy on advisor credibility and persuasiveness. Organiza- 965 tional Behavior and Human Decision Processes, 121:246-255, 2013. doi: 10.1016/j.obhdp. 966 2013.02.001.

13. K. E. See, N. B. Rothman, and Jack B. Soll. Powerful and Unpersuaded: the Implications 968 of Power for Confidence, Advice Taking, and Accuracy. Academy of Management Proceed- 969 ings, 2010(1):1-6, 8 2010. ISSN 0065-0668. doi: 10.5465/AMBPP.2010.54493606.

14. Kelly E. See, Elizabeth W. Morrison, Naomi B. Rothman, and Jack B. Soll. The detrimental 97 effects of power on confidence, advice taking, and accuracy. Organizational Behavior and 972 Human Decision Processes, 116(2):272-285, 11 2011. ISSN 07495978. doi: 10.1016/j. 973 obhdp.2011.07.006.

15. E. S. Gunnar, Reeshad S. Dalal, and Janet A. Sniezek. No decision-maker is an Island: 975 integrating expert advice with information acquisition. Behavioral Decision Making, 19(1): 976 43-60, 2006. doi: 10.1002/bdm.514.

16. David V. Budescu and Adrian K. Rantilla. Confidence in aggregation of expert opinions. 978 Acta Psychologica, 104(3):371-398, 6 2000. ISSN 00016918. doi: 10.1016/S0001-6918(00) 979 00037-8.
17. Jack B. Soll and Albert E. Mannes. Judgmental aggregation strategies depend on whether the self is involved. International Journal of Forecasting, 27(1):81-102, 1 2011. ISSN 01692070. doi: 10.1016/j.jijorecast.2010.05.003.

18. Nigel Harvey and Clare Harries. Effects of judges' forecasting on their later combination of forecasts for the same outcomes. International Journal of Forecasting, 20(3):391-409, 7 2004. ISSN 01692070. doi: 10.1016/j.ijforecast.2003.09.012.

19. Ilan Yaniv and Maxim Milyavsky. Using advice from multiple sources to revise and improve judgments. Organizational Behavior and Human Decision ..., 103:104-120, 2007. doi: 10.1016/j.obhdp.2006.05.006

20. Ilan Yaniv. Receiving other people's advice: Influence and benefit. Organizational Behavior and Human Decision Processes, 93(1):1-13, 1 2004. ISSN 07495978. doi: 10.1016/j. obhdp.2003.08.002.

21. Thomas Schultze, Anne-Fernandine Rakotoarisoa, and Stefan Schulz-Hardt. Effects of distance between initial estimates and advice on advice utilization. Judgment and Decision Making2, 10(2):144-171, 2015.

22. Leonhard Schilbach, Bert Timmermans, Vasudevi Reddy, Alan Costall, Gary Bente, Tobias Schlicht, and Kai Vogeley. Toward a second-person neuroscience. Behavioral and Brain Sciences, 36(04):393-414, 8 2013. ISSN 0140-525X. doi: 10.1017/S0140525X12000660.

23. Leonhard Schilbach. On the relationship of online and offline social cognition. Frontiers in Human Neuroscience, 8, 5 2014. ISSN 1662-5161. doi: 10.3389/fnhum.2014.00278.

24. G. Dumas, G. C. de Guzman, E. Tognoli, and J. a. S. Kelso. The human dynamic clamp as a paradigm for social interaction. Proceedings of the National Academy of Sciences, pages 3726-3734, 8 2014. ISSN 0027-8424. doi: 10.1073/pnas.1407486111.

25. Malika Auvray, Charles Lenay, and John Stewart. Perceptual interactions in a minimalist virtual environment. New Ideas in Psychology, 27(1):32-47, 4 2009. ISSN 0732118X. doi: 10.1016/j.newideapsych.2007.12.002.

26. Jérémie Mattout. Brain-Computer Interfaces: A Neuroscience Paradigm of Social Interaction? A Matter of Perspective. Frontiers in Human Neuroscience, 6(114), 2012. ISSN 1662-5161. doi: 10.3389/fnhum.2012.00114.

27. Ali Mahmoodi, Dan Bang, Majid Nili Ahmadabadi, and Bahador Bahrami. Learning to Make Collective Decisions: The Impact of Confidence Escalation. PLoS ONE, 8(12):e81195, 12 2013. ISSN 1932-6203. doi: 10.1371/journal.pone.0081195.

28. Riccardo Fusaroli, Bahador Bahrami, Karsten Olsen, Andreas Roepstorff, Geraint Rees, Chris Frith, and Kristian Tylén. Coming to Terms: Quantifying the Benefits of Linguistic Coordination. Psychological science, online Jul:1-9, 7 2012. ISSN 1467-9280. doi: 10. $1177 / 0956797612436816$

29. David G. Myers and Helmut Lamm. The group polarization phenomenon. Psychological Bulletin, 83(4):602-627, 1976. ISSN 0033-2909. doi: 10.1037/0033-2909.83.4.602.

30. Serge Moscovici and Marisa Zavalloni. The group as a polarizer of attitudes. Journal of Personality and Social Psychology, 12(2):125-135, 1969.

31. Norbert L. Kerr and R. Scott Tindale. Group Performance and Decision Making. Annual Review of Psychology, 55(1):623-655, 2 2004. ISSN 0066-4308. doi: 10.1146/annurev. psych.55.090902.142009.

32. Annika Boldt and Nick Yeung. Shared Neural Markers of Decision Confidence and Error Detection. Journal of Neuroscience, 35(8):3478-3484, 2 2015. ISSN 0270-6474. doi: 10.1523/JNEUROSCI.0797-14.2015.

33. Nancy R. Buchan, Rachel T.A. Croson, and Sara Solnick. Trust and gender: An examination of behavior and beliefs in the Investment Game. Journal of Economic Behavior \& Organization, 68(3-4):466-476, 12 2008. ISSN 01672681. doi: 10.1016/j.jebo.2007.10.006.

34. Ali Mahmoodi, Dan Bang, Karsten Olsen, Yuanyuan Aimee Zhao, Zhenhao Shi, Kristina Broberg, Shervin Safavi, Shihui Han, Majid Nili Ahmadabadi, Chris D. Frith, Andreas Roepstorff, Geraint Rees, and Bahador Bahrami. Equality bias impairs collective decision-making across cultures. Proceedings of the National Academy of Sciences, page 201421692, 2015. ISSN 0027-8424. doi: 10.1073/pnas.1421692112.

35. Bernhard Treutwein. Adaptive Psychophysical Procedures. Vision Research, 35(17):25032522, 1995

36. Navindra Persaud, Peter McLeod, and Alan Cowey. Post-decision wagering objectively measures awareness. Nature neuroscience, 10(2):257-61, 2 2007. ISSN 1097-6256. doi: $10.1038 / \mathrm{nn} 1840$.

37. Colin W G Clifford, Ehsan Arabzadeh, and Justin a Harris. Getting technical about awareness. Trends in cognitive sciences, 12(2):54-8, 3 2008. ISSN 1364-6613. doi: 10.1016/j.tics.2007.11.009.

38. Stephen M Fleming and Raymond J Dolan. Effects of loss aversion on post-decision wagering: implications for measures of awareness. Consciousness and cognition, 19(1):352-63, 3 2010. ISSN 1090-2376. doi: 10.1016/j.concog.2009.11.002.

39. Benedetto De Martino, Colin F Camerer, and Ralph Adolphs. Amygdala damage eliminates monetary loss aversion. Proceedings of the National Academy of Sciences of the United States of America, 107(8):3788-92, 2 2010. ISSN 1091-6490. doi: 10.1073/pnas. 0910230107.

40. Niccolo Pescetelli, Geraint Rees, and Bahador Bahrami. The perceptual and social components of metacognition. Journal of Experimental Psychology: General, 145(8):949-965, 2016. ISSN 1939-2222. doi: 10.1037/xge0000180.

41. Dan Bang, Riccardo Fusaroli, Kristian Tylén, Karsten Olsen, Peter E Latham, Jennifer Y.F. Lau, Andreas Roepstorff, Geraint Rees, Chris D Frith, and Bahador Bahrami. Does interaction matter? Testing whether a confidence heuristic can replace interaction in collective decision-making. Consciousness and Cognition, 26:13-23, 5 2014. ISSN 10538100. doi: 10.1016/j.concog.2014.02.002

42. Alexandre Pouget, Jeffrey M Beck, Wei Ji Ma, and Peter E Latham. Probabilistic brains: knowns and unknowns. Nature neuroscience, 16(9), 2013. ISSN 1546-1726. doi: 10.1038/ nn.3495.

43. Florent Meyniel, Daniel Schlunegger, and Stanislas Dehaene. The Sense of Confidence during Probabilistic Learning: A Normative Account. PLOS Computational Biology, 11(6): e1004305, 6 2015. ISSN 1553-7358. doi: 10.1371/journal.pcbi.1004305.

44. Wei Ji Ma and Mehrdad Jazayeri. Neural Coding of Uncertainty and Probability. Annual review of neuroscience, 37:205-220, 2014. ISSN 1545-4126. doi: 10.1146/ annurev-neuro-071013-014017. 
45. Adam J. L. Harris, Ulrike Hahn, Jens K. Madsen, and Anne S. Hsu. The Appeal to Expert Opinion: Quantitative Support for a Bayesian Network Approach. Cognitive Science, 40(6) 1496-1533, 8 2016. ISSN 03640213. doi: 10.1111/cogs.12276.

46. R.S. Nickerson. Confirmation bias: a ubiquitous phenomenon in many guises. Review of general psychology, 2:175-220, 1998.

47. Jack B Soll and Richard P Larrick. Strategies for revising judgment: how (and how well) people use others' opinions. Journal of experimental psychology: Learning, memory, and cognition, 35(3):780-805, 5 2009. ISSN 0278-7393. doi: 10.1037/a0015145.

48. Jan Lorenz, Heiko Rauhut, Frank Schweitzer, and Dirk Helbing. How social influence can undermine the wisdom of crowd effect. Proceedings of the National Academy of Sciences of the United States of America, 108(22):9020-5, 5 2011. ISSN 1091-6490. doi: 10.1073/ pnas. 1008636108 .

49. J. Scott Armstrong. Principles of forecasting: a handbook for researchers and practitioners. Kluwer Academic, Norwell, MA, 2001.

50. Stephen M. Fleming and Hakwan C. Lau. How to measure metacognition. Frontiers in Human Neuroscience, 8, 7 2014. ISSN 1662-5161. doi: 10.3389/fnhum.2014.00443.

51. J. A. Sniezek and Timothy Buckley. Social influence in the advisor-judge relationship. In Annual meeting of the Judgment and Decision Making Societ, Atlanta, Georgia, 1989.

52. Gerd Gigerenzer, P. Todd, and ABC Research group. Simple Heuristics that Make us Smart. Oxford University Press, Oxford, 1999.

53. Ralph Hertwig and Gerd Gigerenzer. The 'Conjunction Fallacy' Revisited: How Intelligent Inferences Look Like Reasoning Errors. Journal of Behavioral Decision Making, 12:275205, 1999 .

54. Gerd Gigerenzer, Ulrich Hoffrage, and Heinz Kleinbölting. Probabilistic mental models: A Brunswikian theory of confidence. Psychological Review, 98(4):506-528, 1991. ISSN 0033-295X. doi: 10.1037/0033-295X.98.4.506.

55. Benedetto De Martino, John P. O'Doherty, Debajyoti Ray, Peter Bossaerts, and Colin Camerer. In the Mind of the Market: Theory of Mind Biases Value Computation during Financial Bubbles. Neuron, 79(6):1222-1231, 9 2013. ISSN 08966273. doi: 10.1016/j. neuron.2013.07.003.

56. Charles Mackay. Extraordinary Popular Delusions and the Madness of Crowds. Wordsworth Edition Limited, Ware, UK, wordsworth edition, 1841.

57. Cass R. Sunstein. Republic.com. Princeton University Press, 2001. ISBN 0691095892.

58. Lorien Jasny, Joseph Waggle, and Dana R. Fisher. An empirical examination of echo chambers in US climate policy networks. Nature Climate Change, 5(8):782-786, 5 2015. ISSN 1758-678X. doi: 10.1038/nclimate2666.

59. Asher Koriat. When are two heads better than one and why? Science (New York, N.Y.) 336(6079):360-2, 4 2012. ISSN 1095-9203. doi: 10.1126/science.1216549.

60. Albert E Mannes, Jack B Soll, and Richard P Larrick. The wisdom of select crowds. Journa of personality and social psychology, 107(2):276-99, 8 2014. ISSN 1939-1315. doi: 10. 1037/a0036677. 